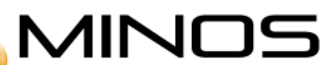

Centre of Excellence for Nuclear Materials

\title{
Multiscale Modeling of Radiation Hardening in RPV and Austenitic Stainless Steels: from the Atomic to the Continuum Scale
}

\author{
Ghiath MONNET ${ }^{1}$ \\ ${ }^{1}$ EDF - R\&D, Centre Les Renardières, (Moret sur Loing, France)
}

Given the nanometric size of radiation defects, Radiation Induced Hardening $(R I H)$ is a textbook case of multiscale physical phenomena. On the one hand, pure atomic features that cannot be described by elasticity contribute strongly to the dislocation interaction. Individual interactions - depending on temperature, dislocation velocity, defect nature and size - can be characterized only when these feature are fully identified and quantified. On the other hand, $\mathrm{RIH}$ is usually observed at the macroscopic scale, where its technological impact is the deepest. Physically based modeling of RIH must thus integrate materials properties from the atomic level. Since explicit and direct integration is not possible, investigations at intermediate scales associated with appropriate transition methods are thus necessary. Pertinent links are known to be the intragranular and the crystalline aggregate scales. The first one provides constitutive equations controlling plastic flow, including $\mathrm{RIH}$, and the second one delivers the homogenized mechanical behavior.

In this paper, we present recent progress in multiscale modeling of RIH in Reactor Pressure Vessel (RPV) steels and internal steels. We first show that the yield stress prior to irradiation has a nonnegligible effect on the $\mathrm{RIH}$, which is found to superpose quadratically with the forest and precipitate hardening. Then, we show how Dislocation Dynamics (DD) simulations can integrate atomistic results and elaborate constitutive equations of plastic behavior. In the following, we present two typical application of our modeling approach.

In internal steels, RIH at doses of some dpa is known to increase tremendously the yield stress. However, the density and the size of the observed radiation defects - formed mostly of dislocation loops - are found (thanks to DD simulations) to provide weak hardening, even when they are considered of extreme strength. The origin of RIH looked difficult to explain. Recently, Atom Probe Tomography (APT) characterization of radiated internal steels have invariably revealed a large density of nanometric solute clusters, which were identified as segregation on small dislocation loops [1].

On the other hand, atomistic simulations have shown that dislocation interactions with dislocation loops leads very often to absorption, as long as the loop is small enough. These experimental observations coupled to atomistic findings inspired a recent investigation [2] in which dislocation interaction with a large number of small dislocation loops were simulated using the DD technique. Since the outcome of individual interactions is invariably absorption, only collinear dislocation loops (i.e. sharing the same Burgers vector as the mobile dislocation) were considered. Interaction with a pure edge dislocation is found to initiate a collective motion of loops in front of the moving dislocation. In contrast, interaction with a pure screw dislocation leads to a large number of collinear interactions strongly pinning the dislocation by forming many helical turns. The stress increment associated with the presence of these loops is depicted on Fig 1a. Hardening is seen to scale with $\mu b \sqrt{D C}$, where $\mu, b$, $D$ and $C$ are respectively the shear modulus, the Burgers vector, the loop size and density. It is clearly seen that the slop of the straight line is close to 0.5 , representing the average interaction coefficient. This value falls within the values reported in experiment. Consequently, results suggest that RIH in internal steels can be attributed to the so called "black dots", which are frequently observed in experiments, rather than the faulted (or unfaulted) large dislocation loops that can be easily resolved in Transmission electron microscopy.

The second example, deals with the possible hardening induced by solute clusters, frequently observed in RPV steels. Since solute clusters are directly revealed by APT in all RPV steels and 
irradiation conditions, they are suspected to be at the origin of RIH in RPV steels. The main difficulty is that it is not possible to use atomistic simulations to compute their resistance, since they are of unknown structure formed of several elements. It is thus necessary to compute the induced hardening as a function of the shear resistance that can be attributed to them. To do so, DD simulations were used to compute hardening induced by a distribution of precipitates of different size, density and shear resistance [3].
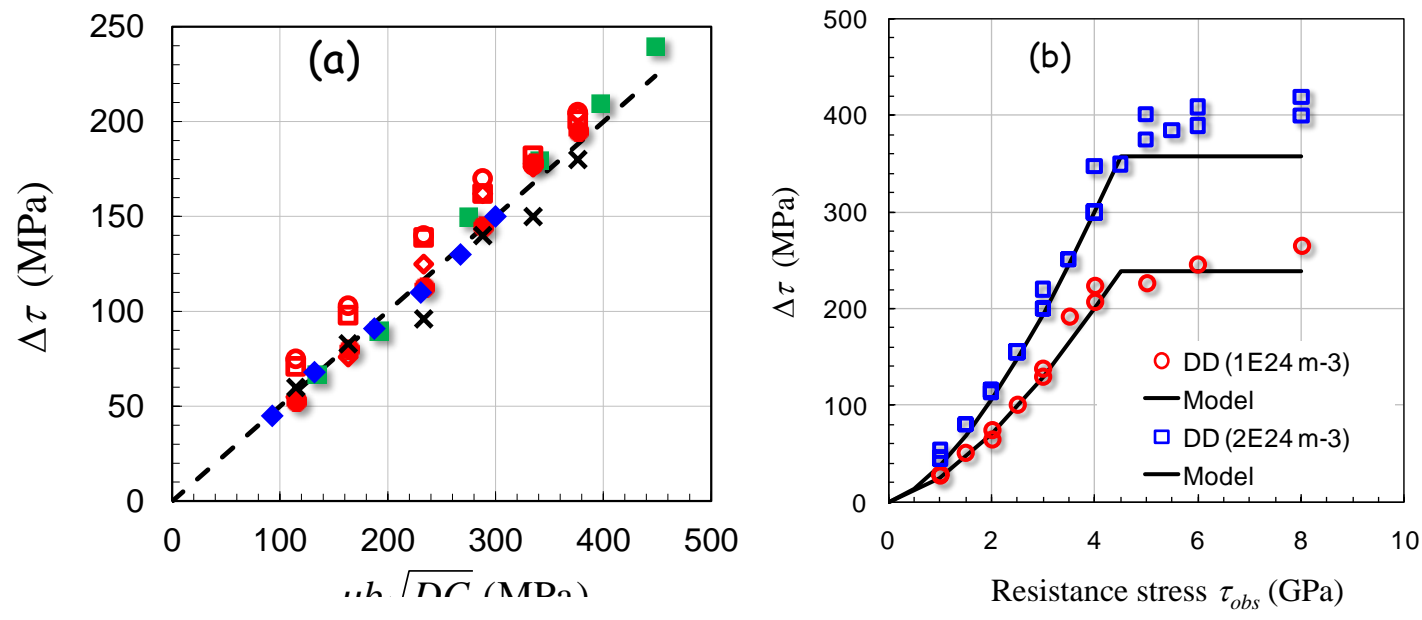

Fig. 1: (a) hardening vs the Orowan stress of collinear dislocation loops [1] and (b) hardening as a function precipitate resistance of $2 \mathrm{~nm}$ and two densities [2]. Curves are predictions of the corresponding constitutive equations.

These investigations have allowed establishing a simple expression predicting precipitation hardening as a function of the precipitate size, density and resistance, as confirmed on Fig. 1b. With this constitutive equation it is possible to provide a good approximation of the solute clusters on the yield stress of RPV steels. Comparison with experiment allows to easily identifying the associated shear resistance.

\section{References}

[1] Edwards, D. J.; Simonen, E. P. \& Bruemmer, S. M. Evolution of fine-scale defects in stainless steels neutron-irradiated at $275^{\circ} \mathrm{C}$ Journal of Nuclear Materials, 2003, 317, 13-31.

[2] Monnet, G. New insights into radiation hardening in face-centered cubic alloys Scripta Materialia, 2015, 100, 24-2.

[3] Monnet, G. Multiscale modeling of precipitation hardening: Application to the Fe-Cr alloys Acta Materialia, 2015, 95, 302-311. 


\section{MatISSE}

Multiscale Modeling of Radiation Hardening in RPV and Austenitic Stainless Steels: from

the Atomic to the Continuum Scale

Ghiath Monnet

$M M C, E D F-R \& D$, Les Renardières, France 


\section{Irradiation induced hardening}

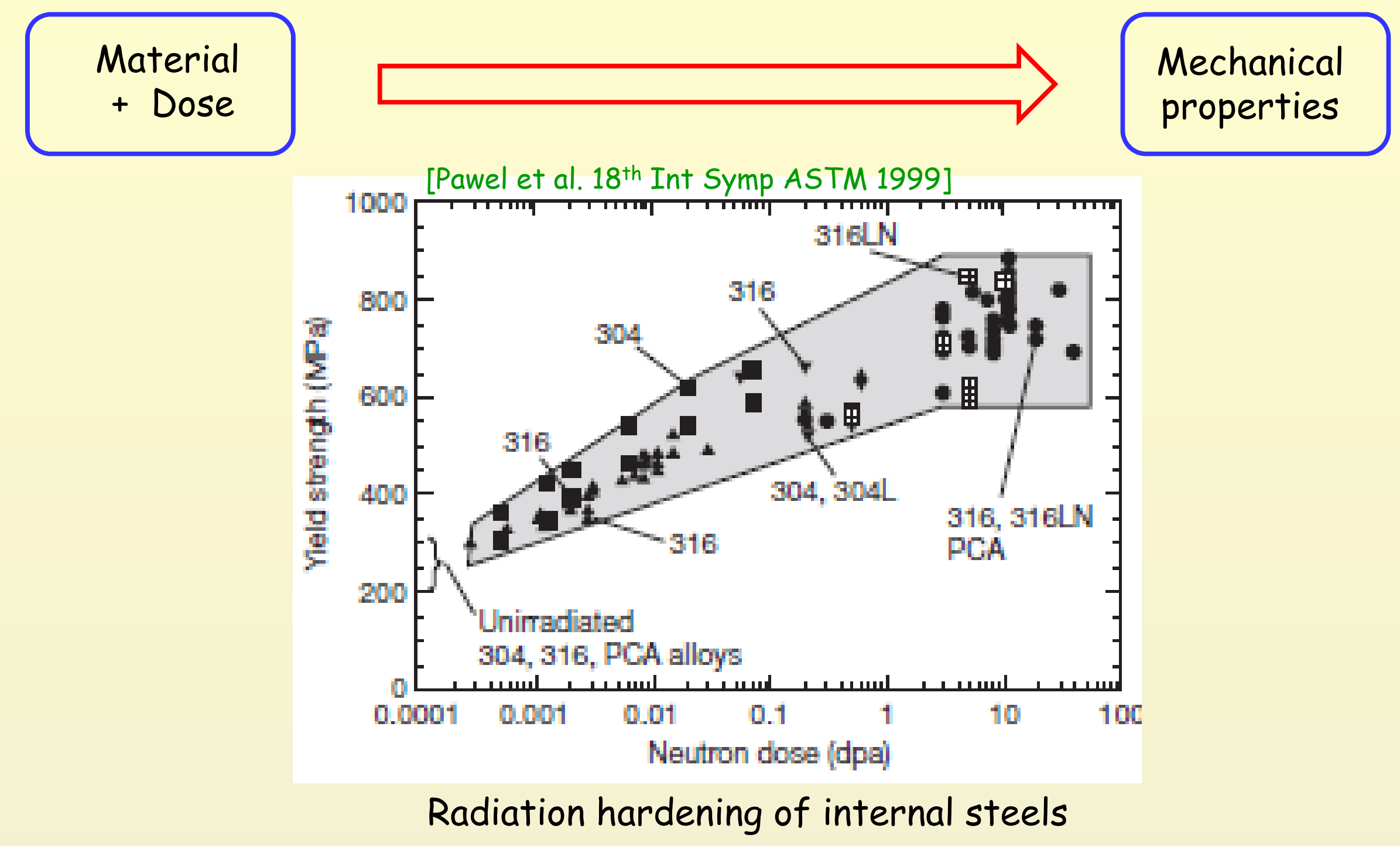

Empirical approach in predicting radiation hardening 


\section{Irradiation microstructures}

316SS, $1 \& 5 \mathrm{dpa}$
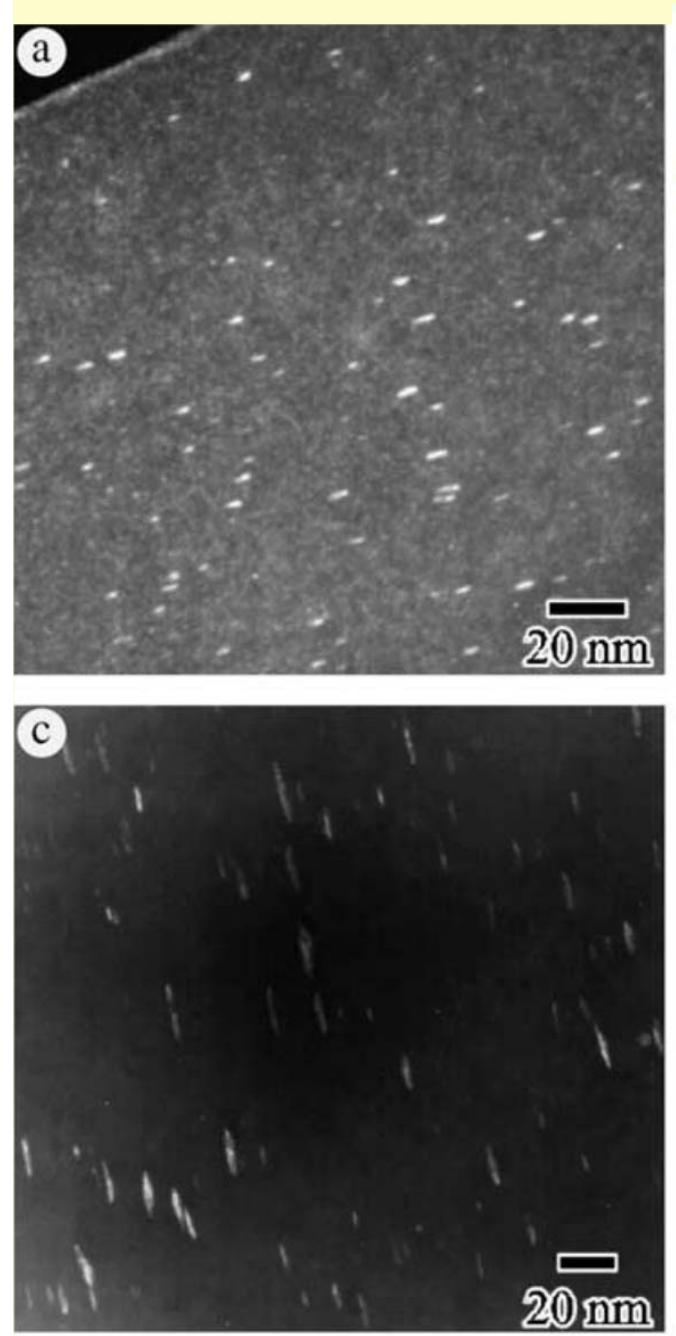

Solute clusters in RPV steels
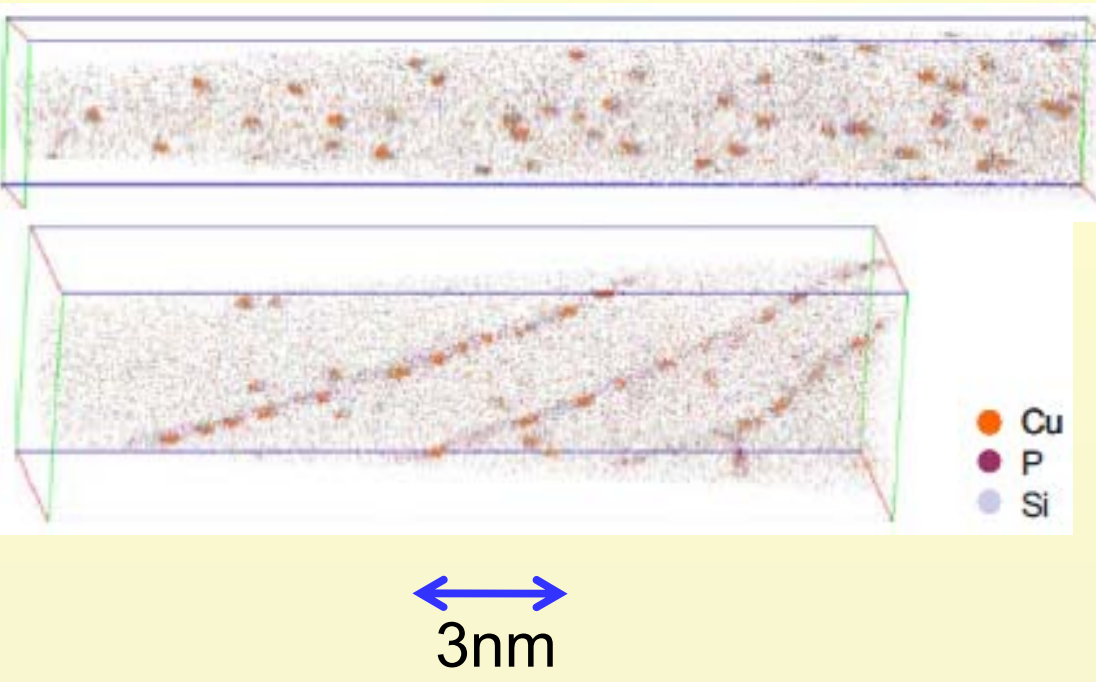

- $\mathrm{Cu}$

Si
0.6 dpa neutron, $\mathrm{Fe}-12 \% \mathrm{Cr}$ alloys

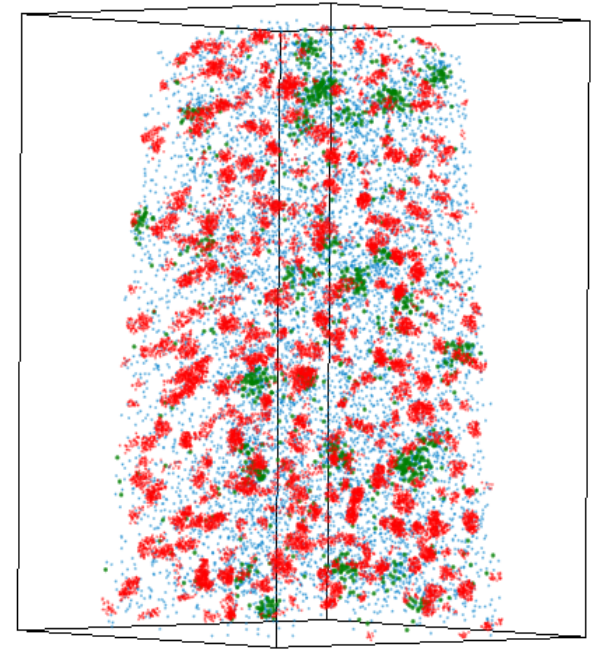

$\mathrm{Cr}$ - and NiSiPCr-clusters

316LN, 3dpa, $\mathrm{T}=363 \mathrm{~K}, 523 \mathrm{~K} \mathbf{1 0 0 \mathrm { nm }}$
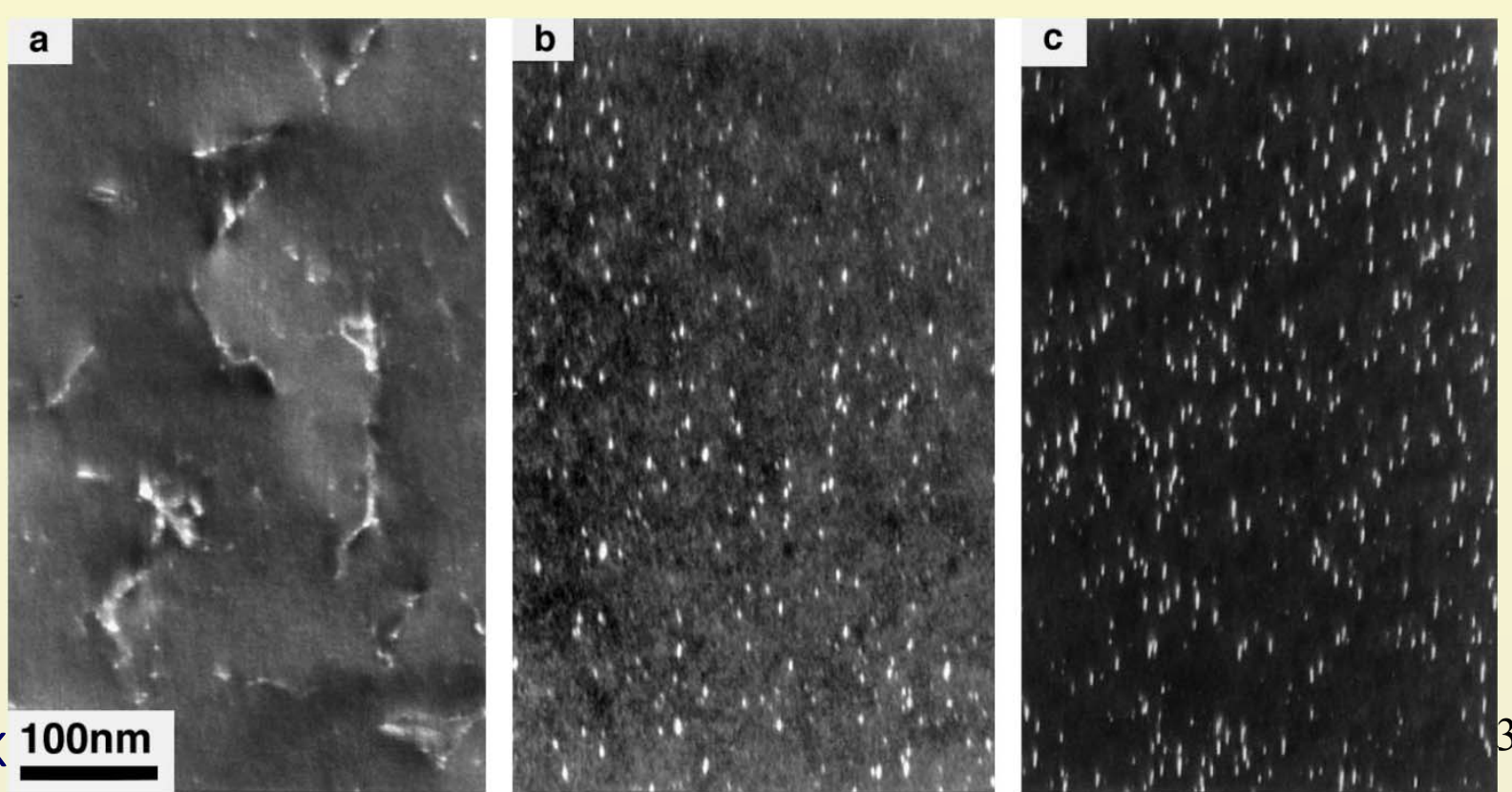


\section{Irradiation induced hardening}

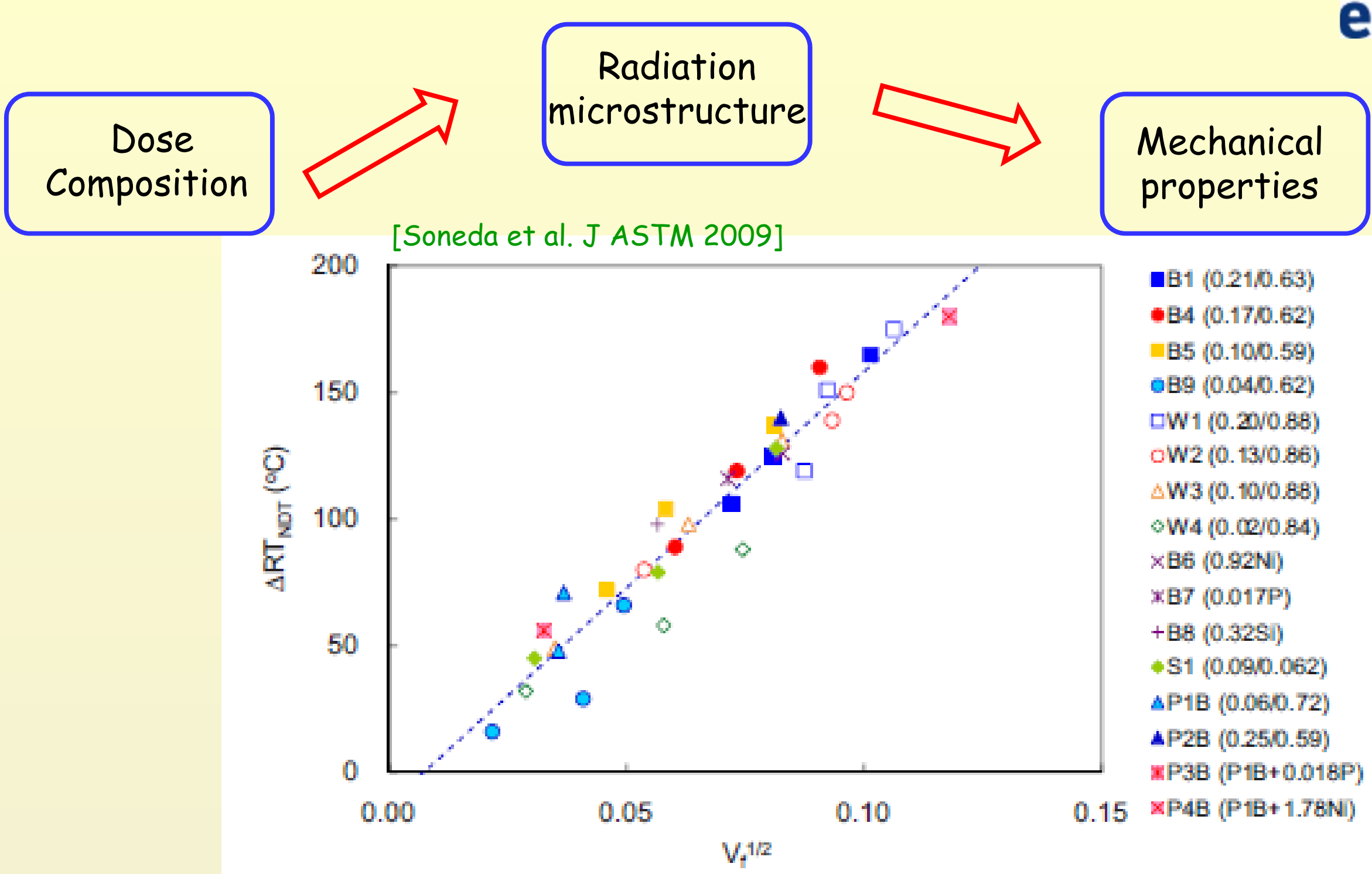

Transition temperature shift in RPV steels

Empirical (through microstructure) approach in predicting radiation 


\section{Multiscale modeling Irradiation induced hardening}

From 2000, European projects on multiscale modeling: (REVE, SIRENA, PERFECT, PERFORM ,..SOTERIA) with partners (EDF, CEA, SCK, ...)

Modeling on physical basis of radiation effects on nuclear materails

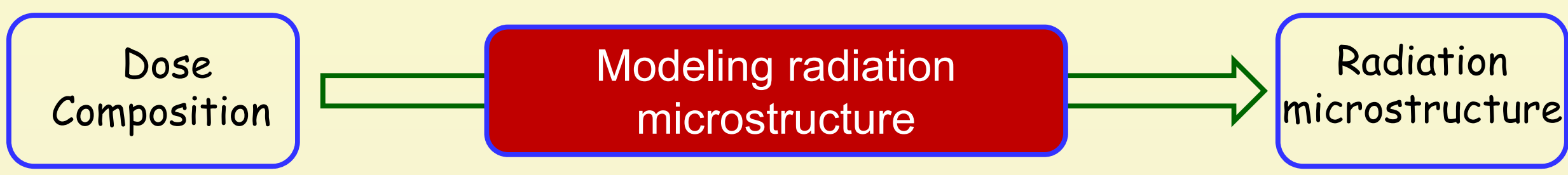

Radiation microstructure

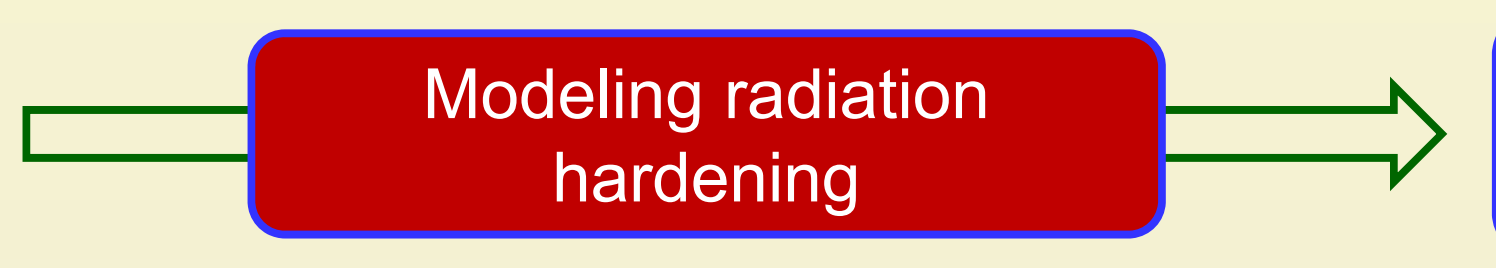

Mechanical properties 


\section{Content}

Two examples of multiscale modeling

\section{Local obstacles}

clusters and precipitates

\section{Extended obstacles}

\section{Atomistic simulation results \\ Transition to the continuum level \\ Prediction hardening at the grain scale}

Conclusions and challenges 


\section{Atomistic results : interaction with solute clusters}

- Simulate dislocation-defect interaction

- Compute the critical stress necessary to unpin from defects

- Evidences of strong interaction with precipitates and voids

- Results depend on the atomistic simulation box

Cu precipitates

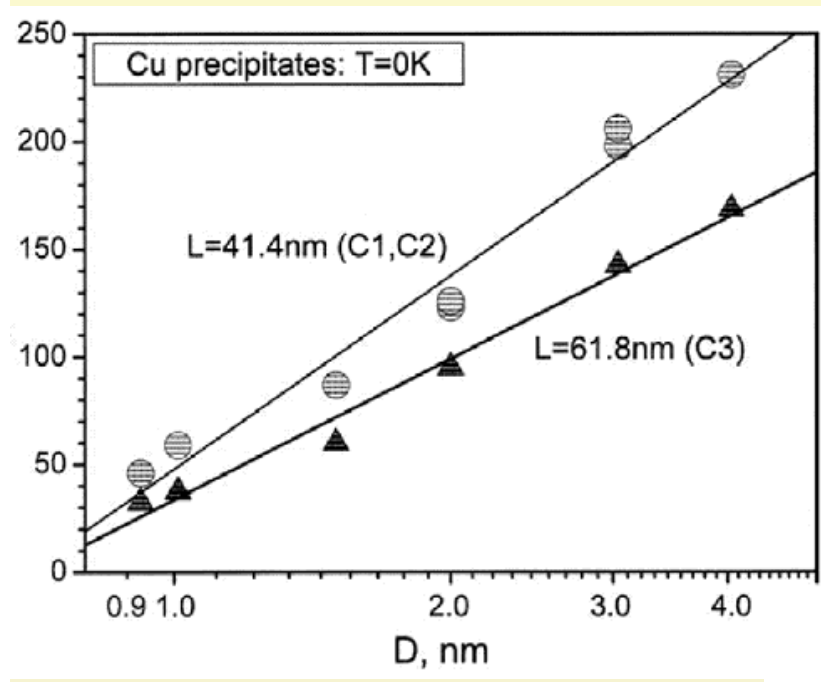

[Osetsky et al. JNM, 2003]

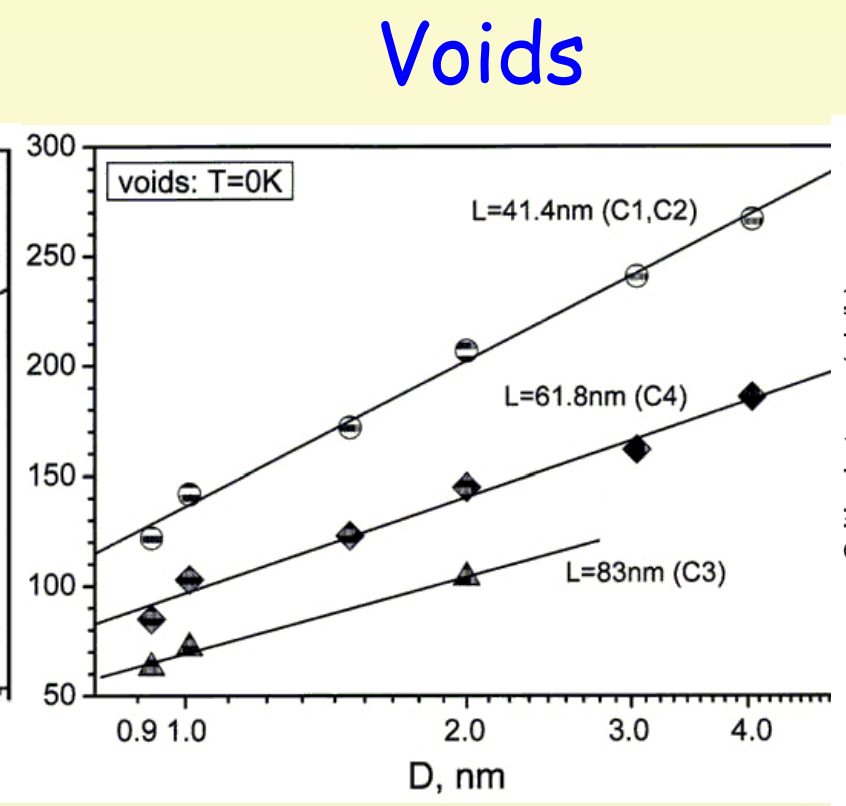

[Osetsky et al Phil Mag 2003]
Cr precipitates

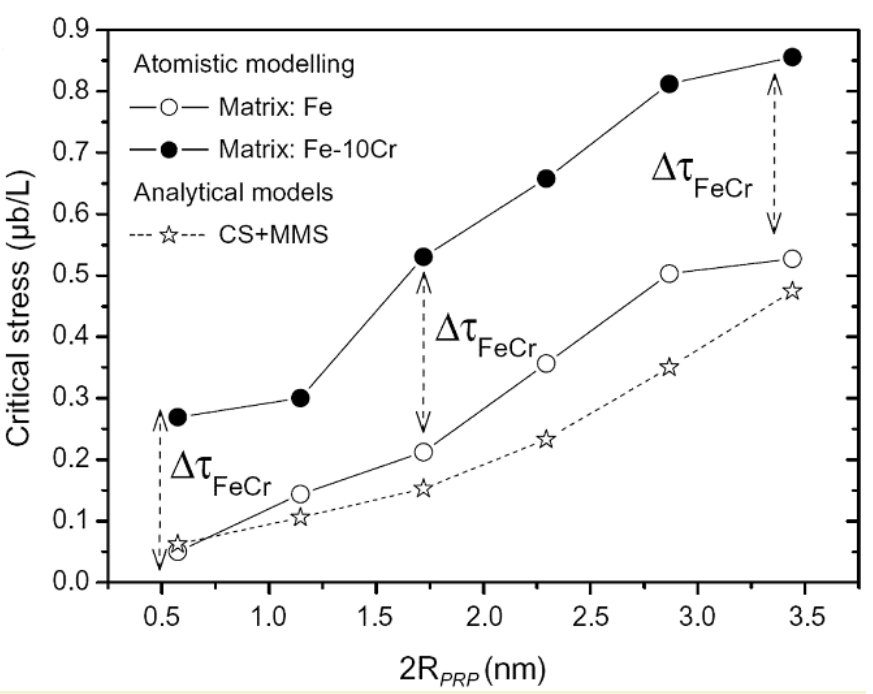

[Terentyev et al. Acta Mat 2008]

How to use these results at larger scale? 


\section{Scale transition to continuum level}

Voids

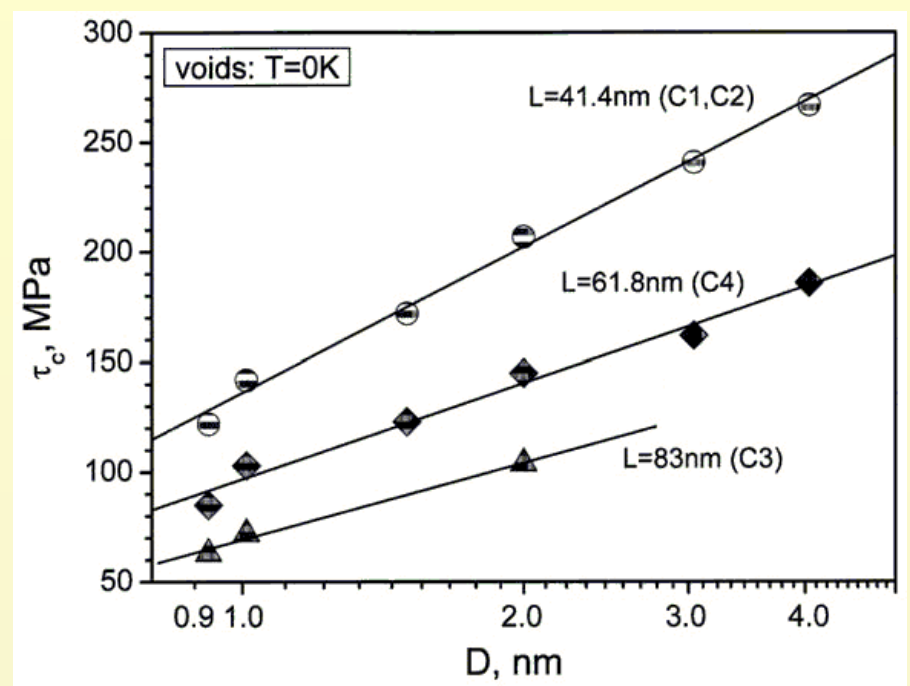

[Osetsky et al Phil Mag 2003]

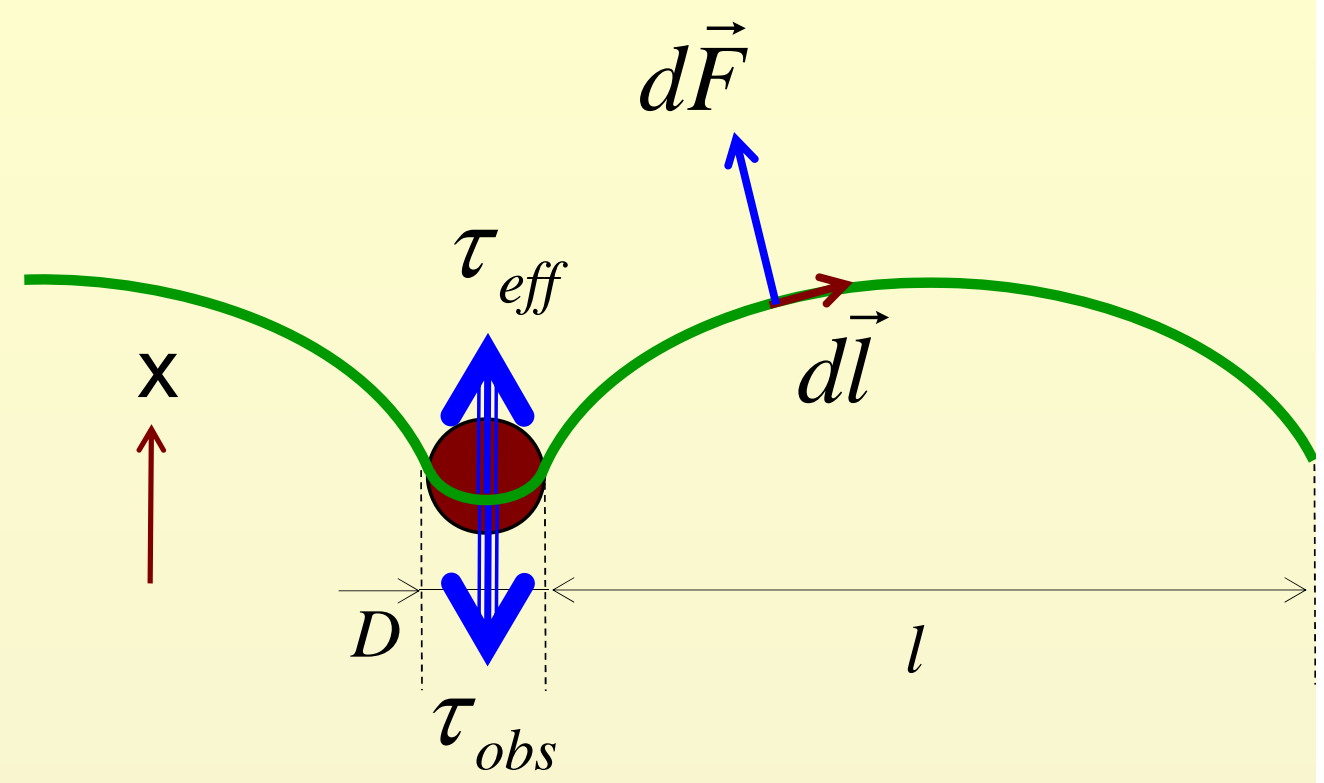

- Mechanical equilibrium $\quad F_{\text {obs }}=\int_{L} b \tau_{a p p} \vec{e}_{x} \cdot d \vec{l}=b \tau_{a p p} L=b \tau_{\text {eff }} D$

- Definition of obstacle resistance $\tau_{o b s}=\frac{L}{D} \tau_{\max }$

- $\tau_{\text {obs }}$ accounts for all interaction mechanisms (lattice, chemical, modulus, etc.)

- $\tau_{\text {obs }}$ can be used in DD simulations 


\section{Scale transition to continuum level}

Application to defects in RPV steels

$$
\tau_{\max }-\tau_{f}=\frac{D}{L} \tau_{o b s}
$$$$
\tau_{\text {voids }}=4.7 \mathrm{GPa}
$$$$
\tau_{\text {Cu prcp }}=2.4 \mathrm{GPa}
$$$$
\tau_{\text {Cr prcp }}=2.1 \mathrm{GPa}
$$

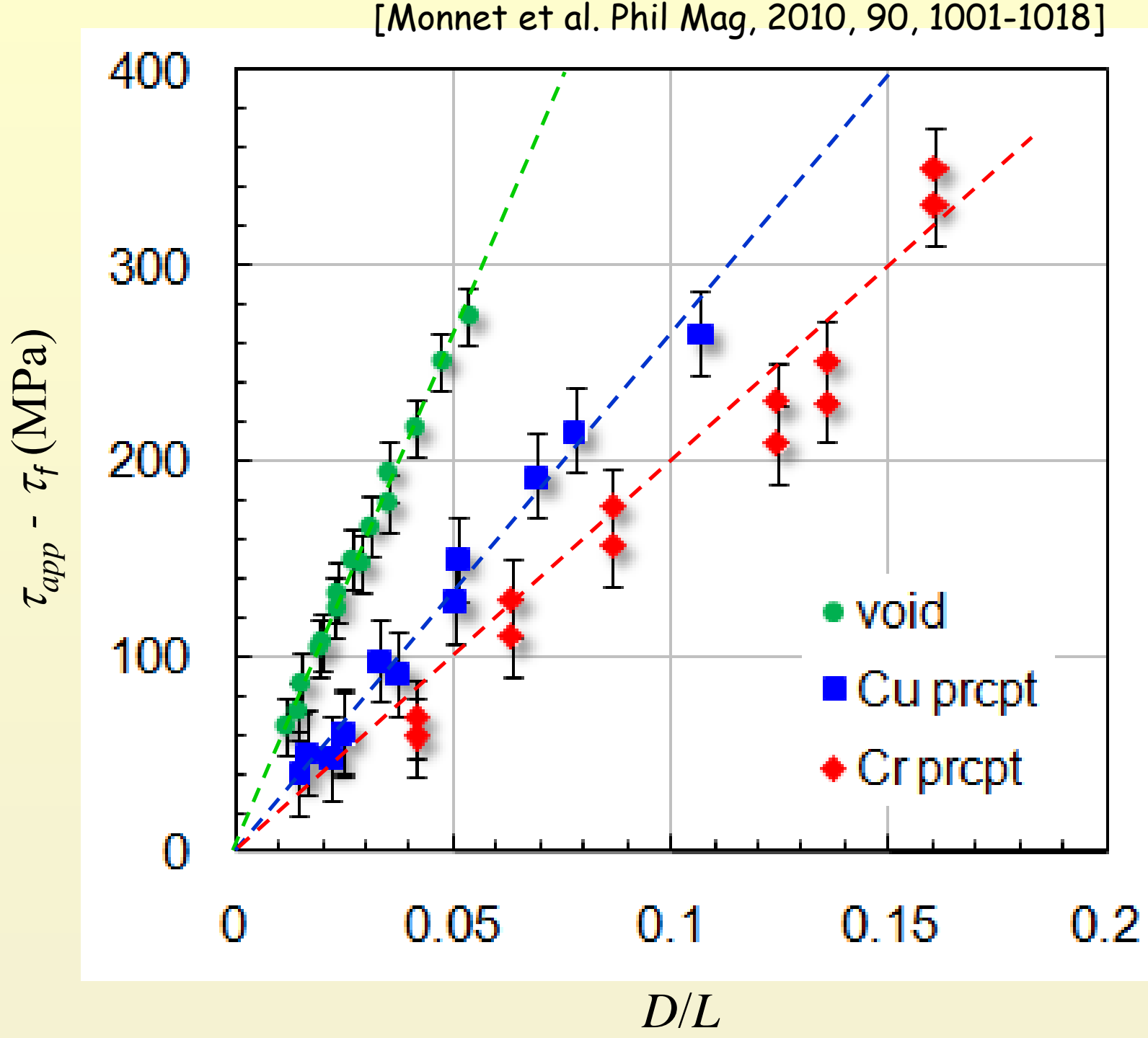




\section{Scale transition to continuum level}

Modeling of dislocation interaction with precipitates

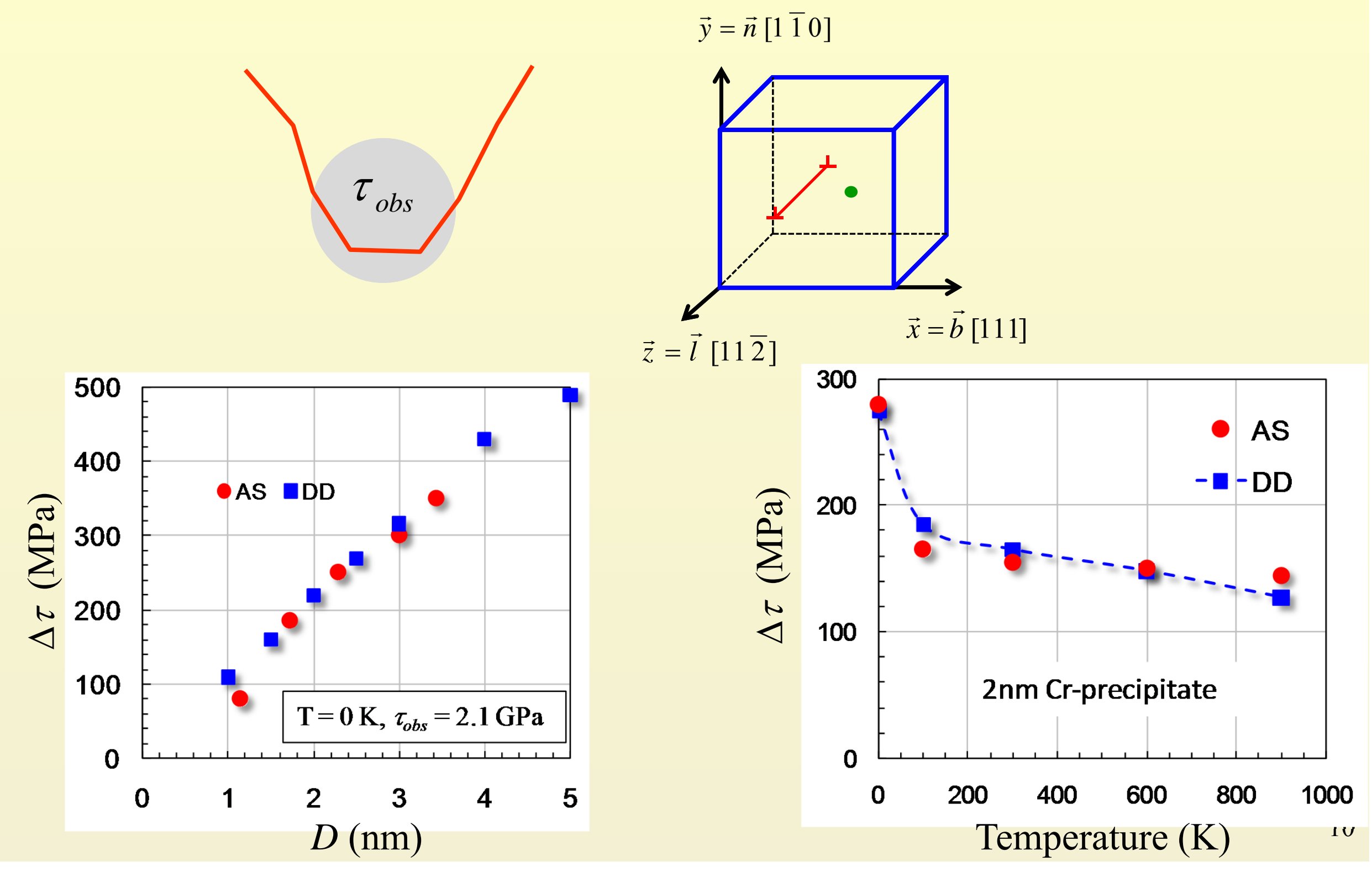




\section{Modeling radiation hardening at the grain scale}

Modeling of dislocation interaction with precipitates

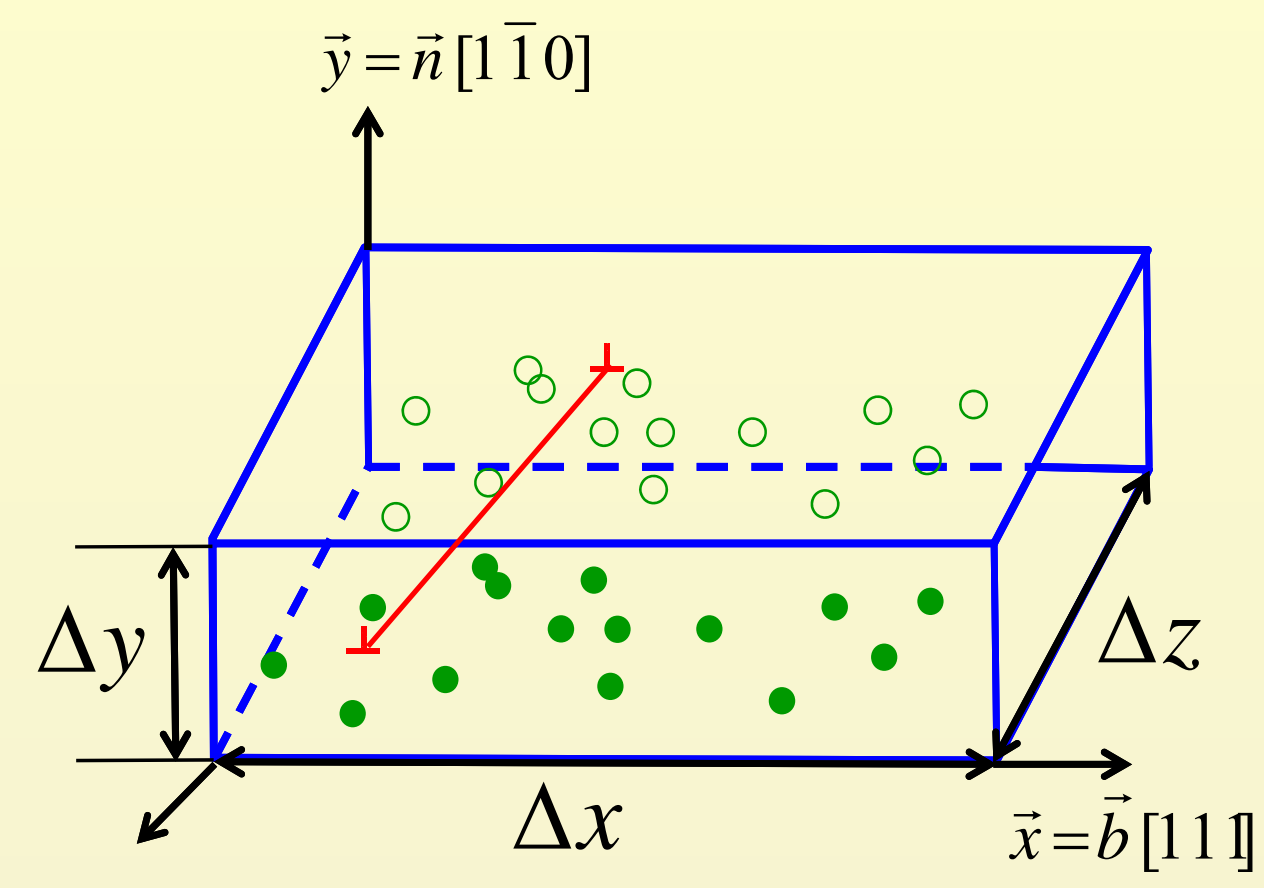

- 20000 precipitates

- Random distribution

- Dislocation length > 20 I

- $D=1,2,4 \mathrm{~nm}$

- $\mathrm{C}=10^{23}-810^{24} \mathrm{~m}^{-3}$

$\overrightarrow{\mathrm{Z}}=\vec{l}[11 \overline{2}]$ 


\section{Modeling radiation hardening at the grain scale}

Simulations of hardening induced by Orowan obstacles

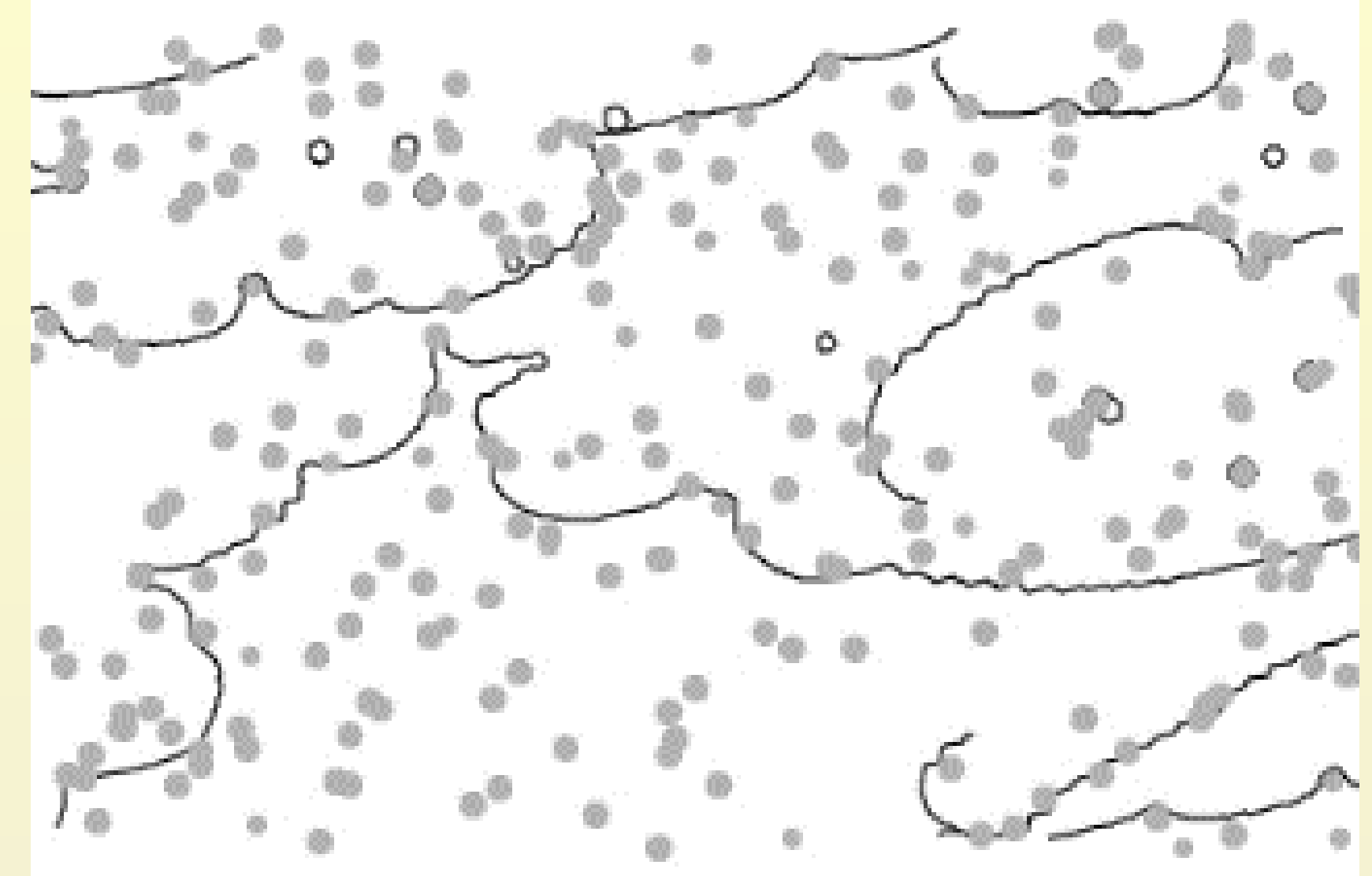




\section{Modeling radiation hardening at the grain scale}

Constitutive equation for hardening induced by local obstacles

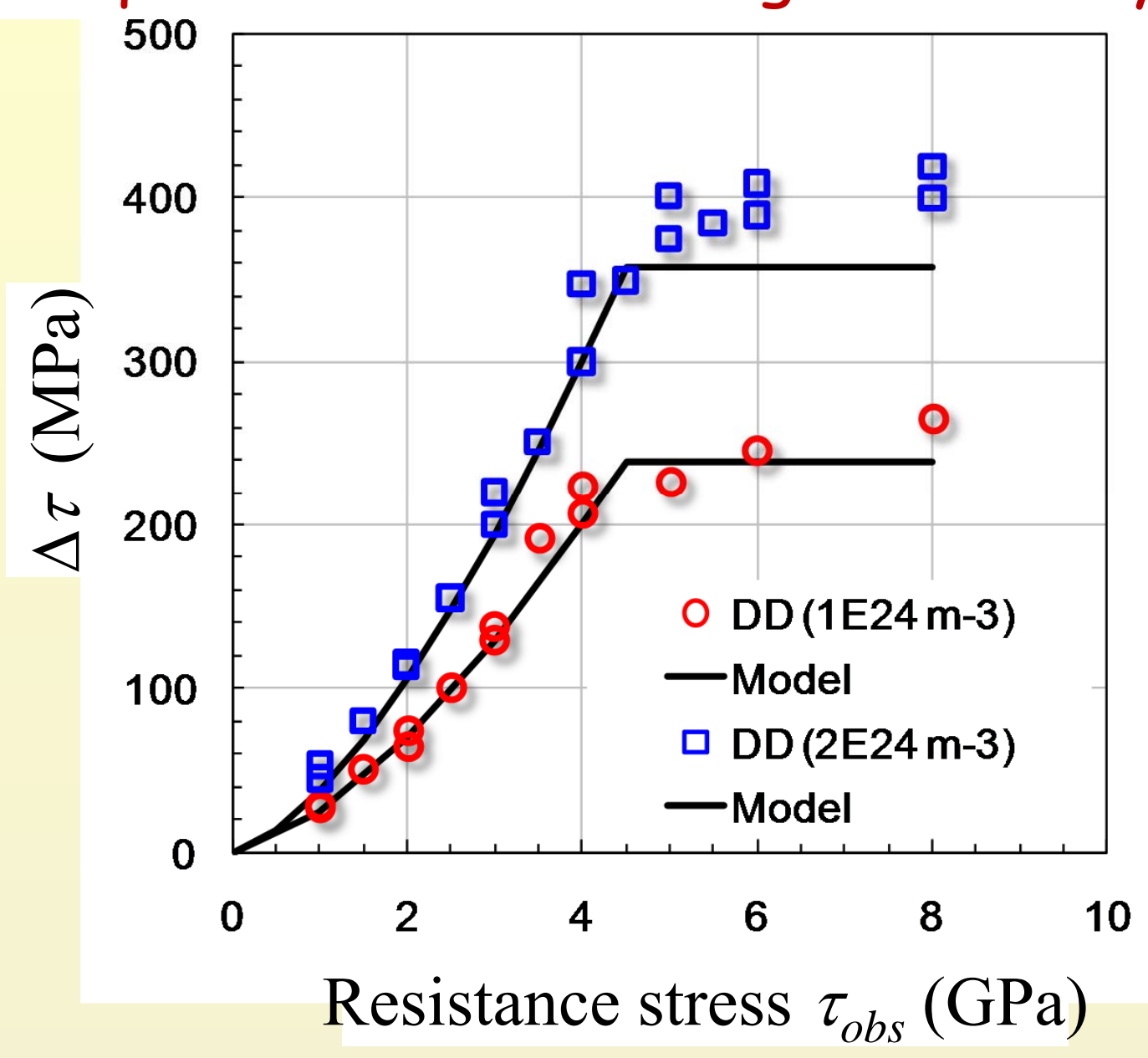

[Monnet, Acta Mat 2015]

$$
\Delta \tau=\left(\frac{\tau_{o b s}}{\tau_{\infty}} \frac{\ln 2 \underline{D}}{\ln 2 l}\right)^{3 / 2} \frac{\mu}{2 \pi l} \ln (2 l), \quad l=\frac{1}{\sqrt{D C}}-D
$$




\section{Atomistic results : interaction with dislocation loops}

Screw vs $\frac{1}{2}[111]$ loop

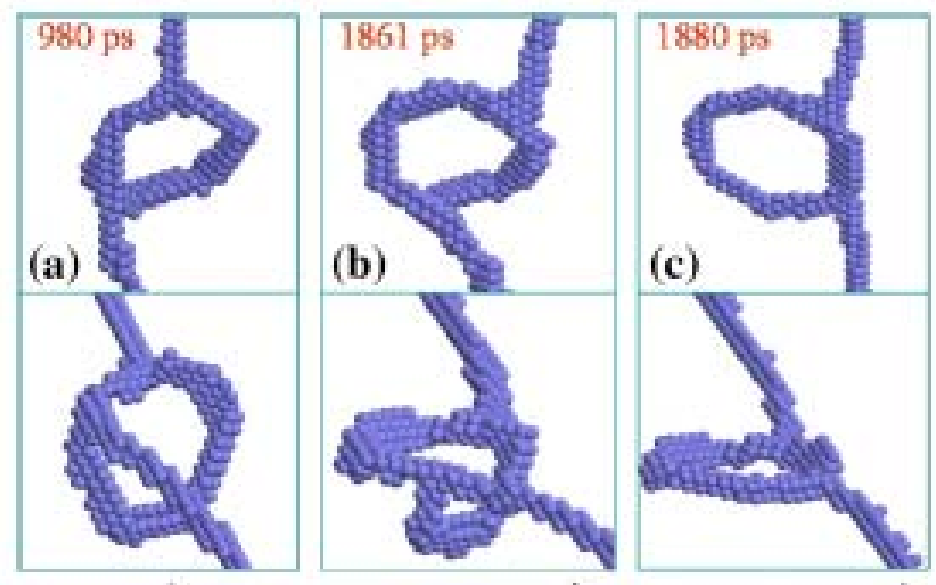

Edge vs [100] loop
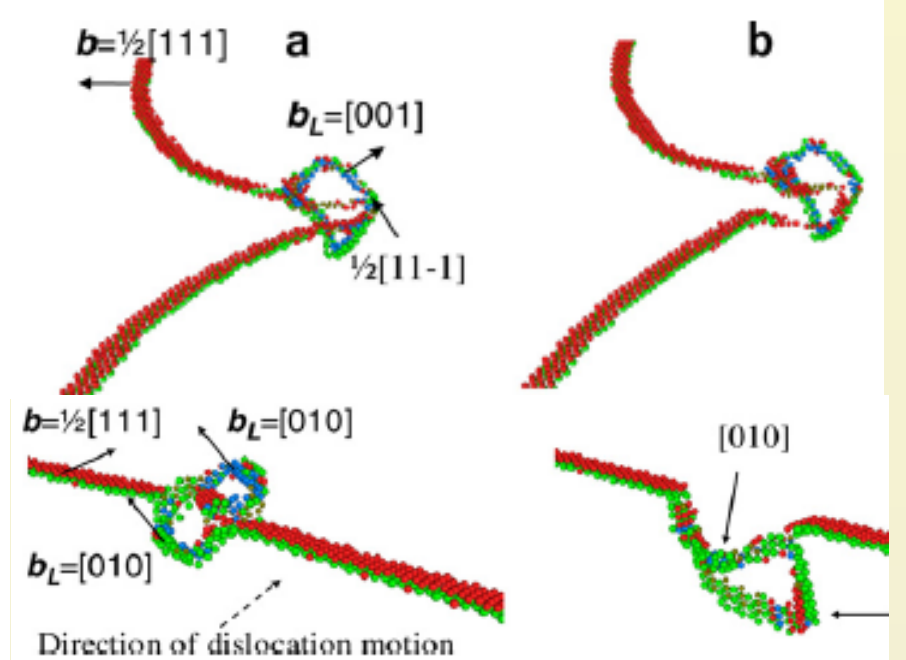

FeNiCr alloys
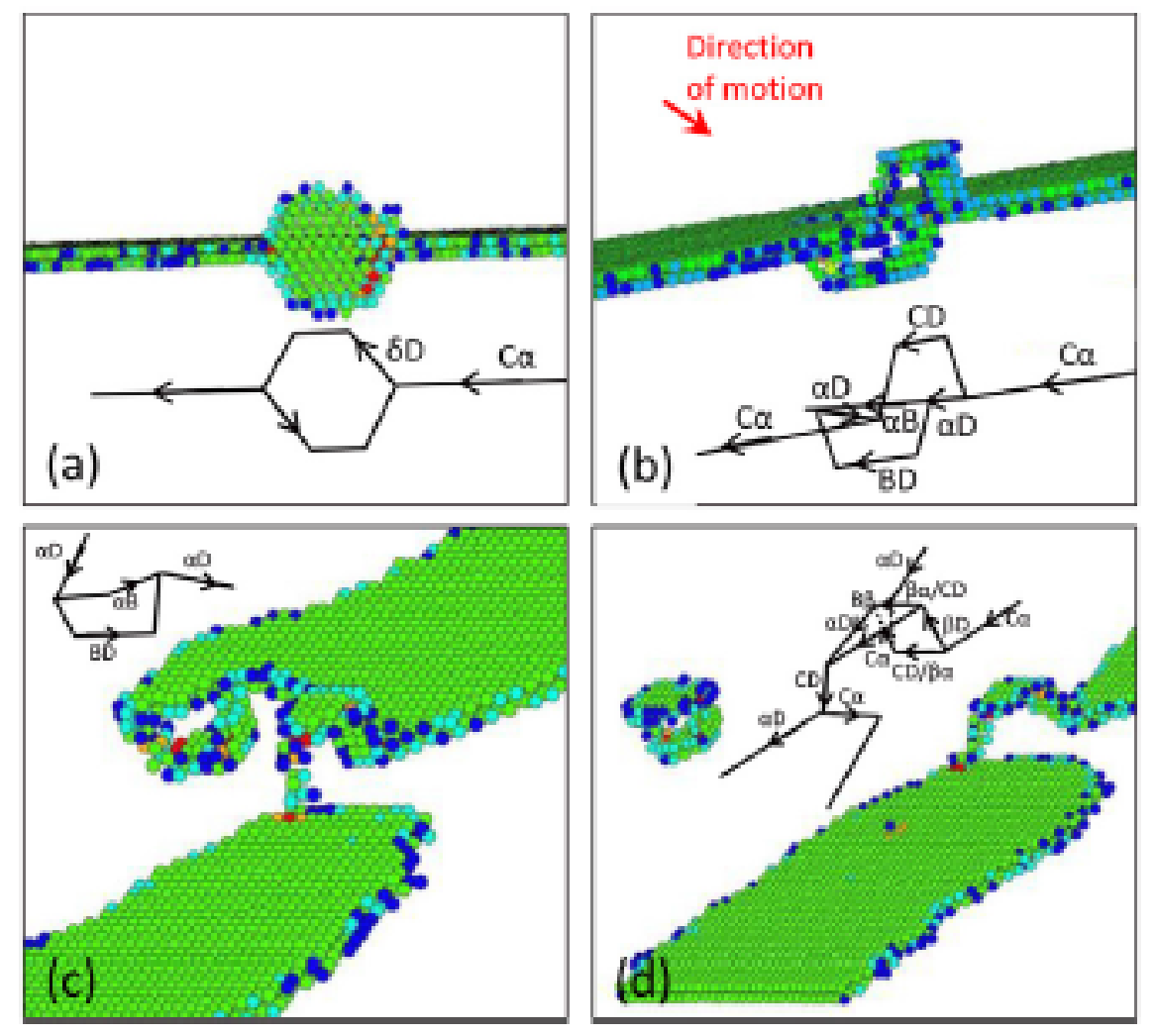

Edge vs Frank loops

[Liu et al. Scripta 2008]

[Terentyev et al. Acta Mat 2008]

[Baudouin et al, JNM 2015] 


\section{Scale transition to continuum level}

Dislocation interaction with loops at the continuum level

Large variety of interaction mechanisms depending on

- Effect of loop size, nature and orientation

- Effect of temperature and strain rate

- Effect of dislocation character

General trends

- Large dislocation loops are sheared

- Small dislocation loops are absorbed 


\section{Modeling radiation hardening at the grain scale}

\section{eDF}
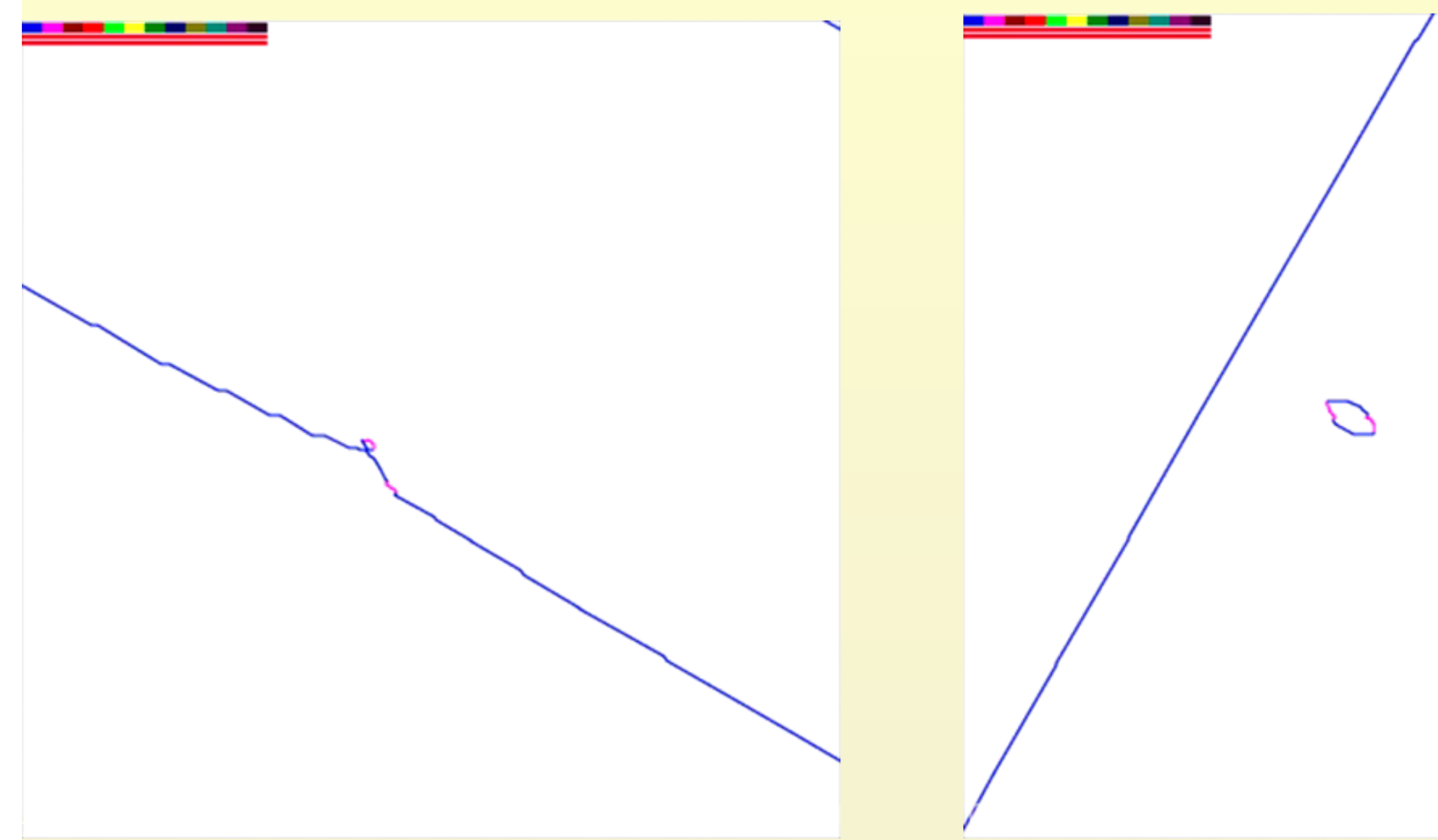

Absorption by edge dislocation

Absorption by screw dislocation 


\section{Modeling radiation hardening at the grain scale}

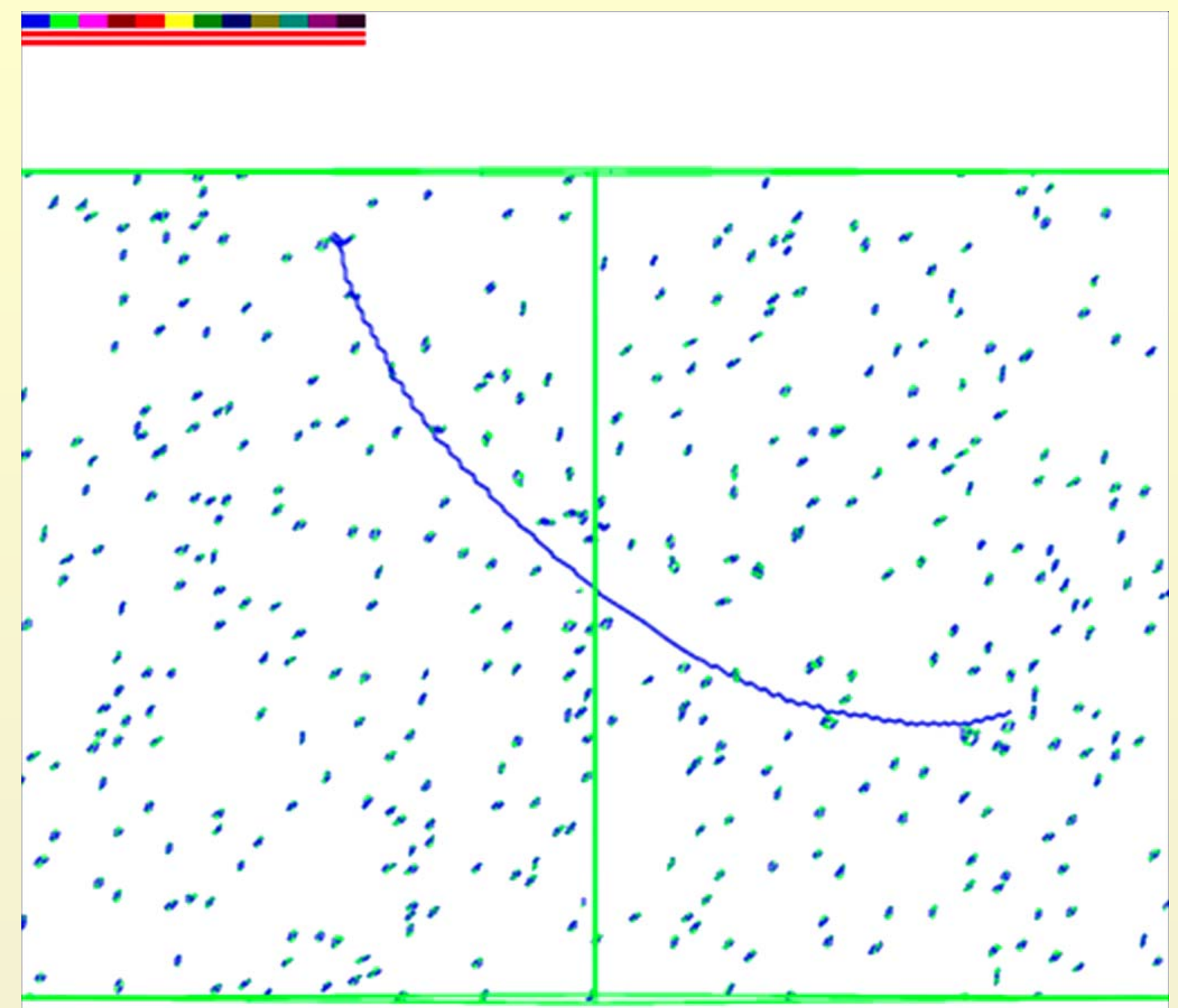




\section{Modeling radiation hardening at the grain scale}

[Monnet, Scripta Mat 2015]

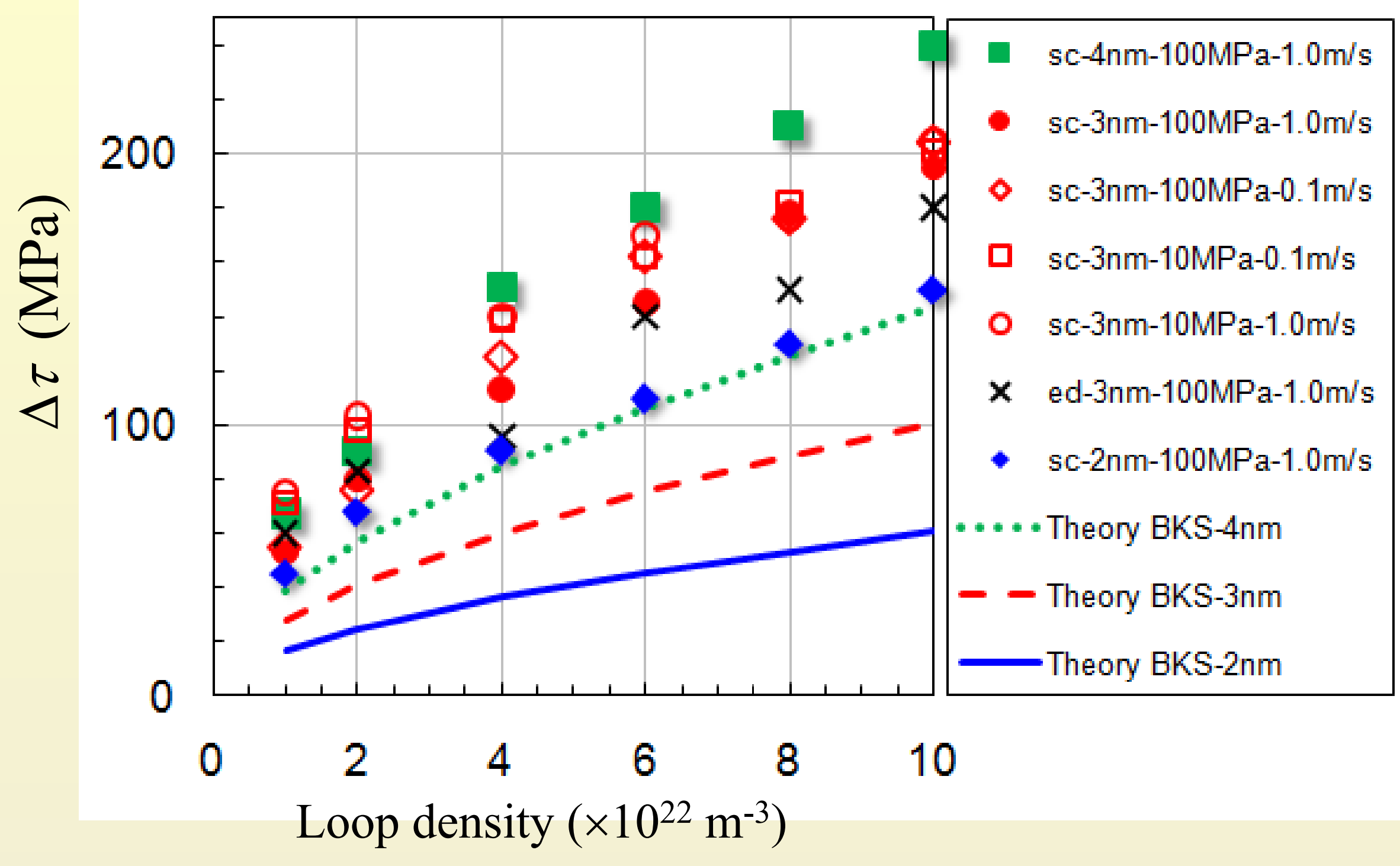




\section{Modeling radiation hardening at the grain scale}

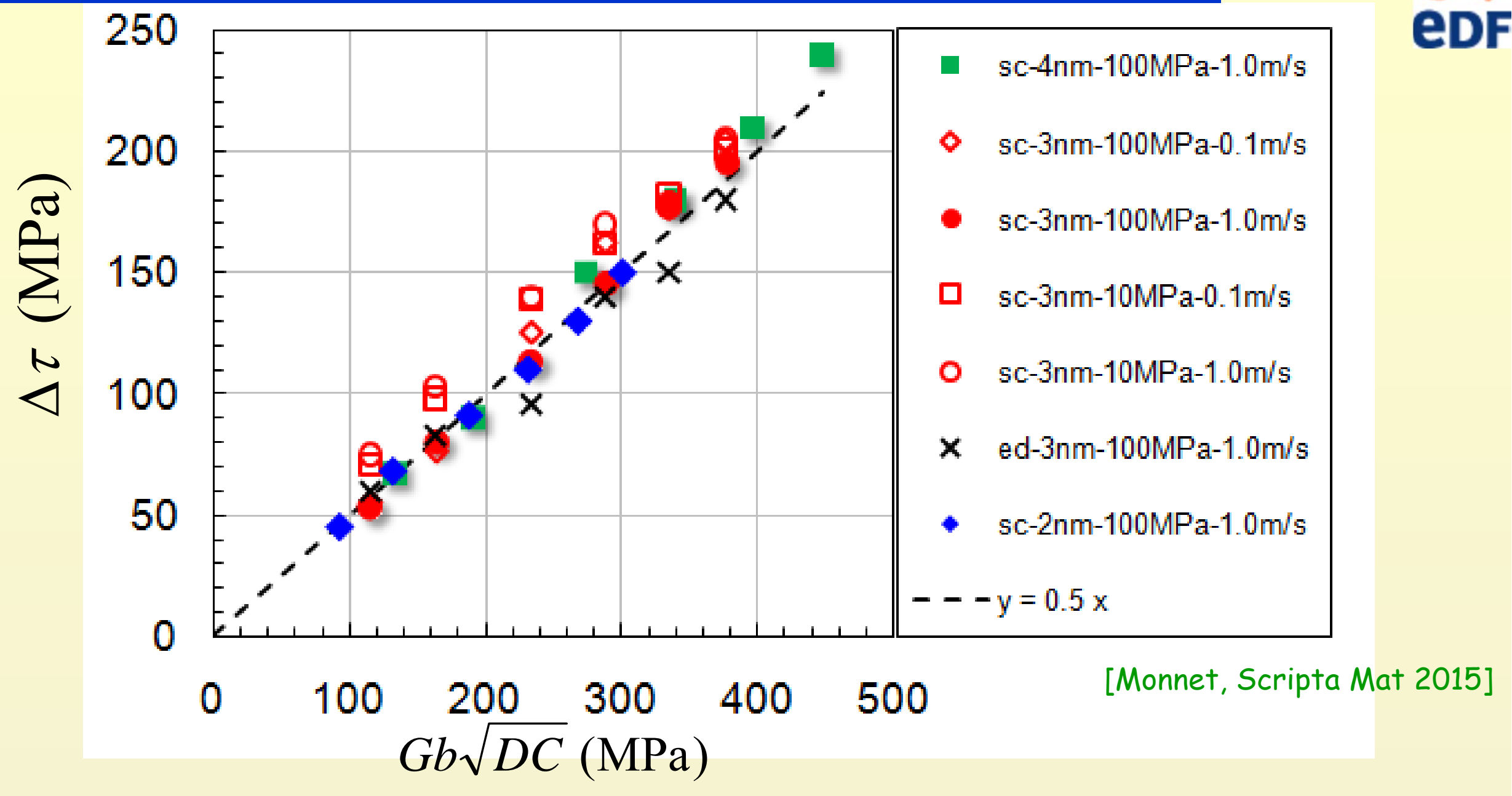

$$
\Delta \tau=0.5 G b \sqrt{D C}
$$$$
\dot{C}^{s}=-\lambda \frac{D}{b} C^{s}\left|\dot{\gamma}^{s}\right|
$$ 


\section{Conclusions \& challenges}

Achievements of multiscale modeling:

- Atomistic simulations of edge dislocation

- Interactions with simple defects ( $C r, C r$ prcp, loops, voids, SFT, etc.)

- Dislocation Dynamics simulations of precipitation hardening

- DD simulations of hardening induced by small loops

- Construction of crystalline laws for RPV and internal steels

\section{Future challenges:}

- Prediction and modeling of radiation microstructure

- Investigating solute segregation effects (decoration, mobility, strength)

- Dislocation interaction with grain boundaries

- Accounting for softening in crystalline laws in macroscopic modeling

- Allowing for mesh-independent strain heterogeneity 